\title{
One-dimensional Orientation Using a Low-cost Inertial Sensor
}

\author{
M. Haid, T. Chobtrong, M. Kamil, E. Günes \\ Competence Center for Applied Sensor System, \\ Darmstadt University of Applied Sciences, \\ Birkenweg 8, 64295 Darmstadt, Germany
}

\begin{abstract}
:
This paper presents one-dimensional attitude estimation using a low-cost gyroscope with help of shaping filter for industry applications. This algorithm is a part of controller module, which will be implemented in an intelligent wrench for automotive manufacturing. The orientation is primary estimated by the signals of a low-cost pinpoint gyroscope. With help of an error model based on Shaping filter method, the optimal 1-D orientation of an object is determined by compensating the estimated orientation with the estimated error. As the results from experiments, the accuracy of this attitude estimation is less than 1 degree within 90 second.
\end{abstract}

Key words: attitude estimation, low-cost gyroscope, Shaping filter, tracking, error model

\section{Introduction}

Currently, most of processes in automotive manufacturing have been carefully controlled and tested to guarantee the quality of the final products. However, many parts of a vehicle still have been assembled without any quality control processes, or with poor control procedures. For example, a worker uses a wrench to fasten a bolt that holds a pulley on an engine. He should fasten the bolt with the right torque. At the same time, the bolt should be rotated within a control range. If the rotating angle of the bolt is out of the control range, it might be that a worker uses a wrong bolt or the bolt is not in the specification. To control this process and improve the quality of automotive assembly processes, it is necessary to develop an intelligent wrench which is able to measure applied torque and a rotating angle of a bolt.

A low-cost pinpoint gyroscope, CRM100 is applied for one-dimensional orientation estimation of the intelligent wrench. Instead of detecting a rotating angle of a fastened bolt, the intelligent wrench detects its own orientation which is the same angle as the rotating angle of the bolt. However, the challenge of this orientation estimation with a low-cost gyroscope is sensor drift problem that generated the accumulated error over time [1]. Moreover, it is difficult to integrate compass sensors as observation sensors, because there are the distortion of magnetic field and magnetic interferences in automotive production line [2].
Theoretically, the orientation of an object attached with a gyroscope, is able to be numerically estimated by integration of angular velocity signals. But, in practice, there is always stochastic sensor drift in the measurement signals from a low-cost gyroscope [3]. Therefore, the error in orientation estimation using low-cost gyroscopes increases over time dramatically, because of the accumulated error for the integration.

To eliminate the effect of sensor drift from attitude estimation, Kalman filter (KF) and variants of Kalman filter is well-known technique to find the optimal attitude from the signals of a low-cost inertial measurement unit (IMU). Foxlin [4] applied extended Kalman Filter (EKF) based on the separated bias Kalman filter method from Friedland in [5]. However, the yaw estimation of this method is depended on the signals from magnetometers as many studies in [6], [7], [8], [9], [10] and [11]. For automotive assembly line, there are many metallic objects that distort the magnetic field. Therefore, using magnetometers for attitude estimation might lead to the inaccuracy of the system. Another problem of EKF, it is wellknown that the behavior of EKF is unpredictable, although it often can be used successfully [12].

Instead of EKF, other studies, such as in [13], successfully developed attitude estimation algorithms based on unscented Kalman Filter (UKF). Although the mathematic model of UKF 


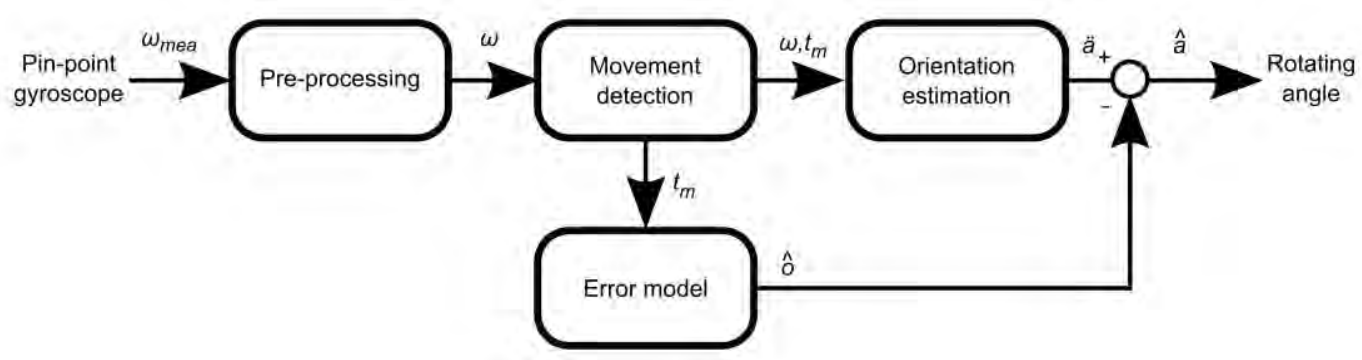

Fig. 1. Algorithm structure of the orientation estimation

is simpler than EKF, because of it is not necessary to calculate Jacobian matrices [14]. The computation time of UKF is greater than $E K F$, and it is not suitable for real-time applications. Interestingly, Rehbinder and $\mathrm{Hu}$ successfully developed the attitude estimation based on switching Kalman Filter [12]. Importantly, the mathematic model of their work is simply and based on complementary Kalman filter and the switching algorithm in [15]. There is the switching algorithm for selecting between static mode and dynamic mode. This technique is really practical for eliminating the effect of lateral acceleration while the object is moving. However, the output of this attitude estimation is just only the optimal roll and pitch angles.

This paper presents the one-dimensional orientation using low-cost gyroscope based on error model technique with help of shaping filter for estimation rotating angle in environment that is not able to use magnetometers.

\section{Algorithm Structure of Orientation Estimation}

There are 4 modules to estimate the orientation of the intelligent wrench, such as Preprocessing module, Movement detection module, Orientation estimation module and Error model module. The overview structure of this orientation estimation is shown in Fig. 1.

The Pre-processing module helps the system to reduce noise of the measurement signal, $\omega_{m e a}$ from a CRM100 pin-point gyroscope from PinPoint, and decrease the number of data using for orientation estimation. This preprocessing module contains algorithms that resample the measurement signal from $1 \mathrm{kHz}$ to $50 \mathrm{~Hz}$ sampling frequency, and filter the signals with low-pass Butterworth filter.

To observe the movement of the intelligent wrench, the Movement-detection module detects the change from static state to dynamic state of the tool. Because there is stochastic noise in the measurement signal, the error from orientation estimation is dramatically reduced when processing the data in short time [3]. Therefore, it is necessary to use the measurement signal only within dynamic state (while rotating a wrench to fasten a bolt).

With the angular velocity signal $\omega$ and the movement time $t_{m}$, the one-dimensional orientation of the wrench is determined by the Orientation-estimation module. In brief, the algorithm in this module is based on Newton's laws of motion.

The Error-model module generates the optimal misalignment, $\hat{o}$, to compensate the estimated orientation, ä. This Error-model module is heuristically designed based-on Shaping filter technique and optimized with the Nelder-Mead Simplex Method in Low Dimensions [16].

As the output of this orientation estimation, the optimal rotating angle, $\hat{a}$ is determined by compensating the estimated orientation ä with the optimal misalignment $\hat{o}$. This information will be sent as to the controller of the intelligent wrench for making a decision whether the fastening is acceptable.

\section{Error Model}

In many cases, the white Gaussian noise model may not adequate to predict all noises in a real system. Practically, an error model is developed based on a mathematic model to generate empirical autocorrelation or power spectral density data as the same as the data from noises of an observed system. A linear timeinvariant system, or shaping filter, provides a model driven by stationary white Gaussian noise which is determined by the power spectral density or autocorrelation of observed data. In other words, if the first and second order statistics of a wide-sense stationary are known, then a Gaussian process with the same first and second order statistics can always be generated via shaping filter [17].

In this paper, the error model for the orientation estimation is heuristically constructed based on the first order Markov model [18]. This error model generates exponentially time-correlated error signal which is statistically similar to the error from drift of the gyroscope. This model is described as $\mathrm{Eq}(1)$, where $x$ is the state of the 
model, $T$ is the time correlation, and $w$ is a Gaussian noise with zero mean.

$x^{\prime}(t)=-(1 / T) x(t)+w(t)$

As proofed in $[X]$, the output from this model is has autocorrelation $\Psi_{\mathrm{xx}}$ as $\mathrm{Eq}(2)$ where $\delta$ is a standard deviation and $\tau$ is a time constant.

$\Psi_{\mathrm{xx}}(\tau)=\delta^{2} e^{\left(-\left.\right|_{\tau} / T\right)}$

To initialize model parameter, the stationary signals from the pin-point gyroscope have been acquired to determine empirical autocorrelation and power spectral density of the signals. By curve-fitting, the initial parameter of $\tau$ is defined. This initial parameter will be used as an initial value for optimizing the error model parameters.

The bias $b$ and misalignment $o$ of the pin-point gyroscope is defined as the state of this error model. The behavior of the misalignment is assumed to be exponentially time-correlated state as the first order Markov model. Therefore, the dynamic equation of the model is constructed as in $\mathrm{Eq}(3)$, where $\beta=1 / \tau$.

$\left[\begin{array}{l}b^{\prime} \\ o^{\prime}\end{array}\right]=\left[\begin{array}{cc}-\beta & 1 \\ 1 & 0\end{array}\right]$

This error model is driven by initial bias and misalignment, $b_{0}$ and $o_{0}$ in order. Both of the initial bias and misalignment will be heuristically optimized with the Nelder-Mead Simplex Method as described in the next section.

\section{Parameter Optimization}

The error-model parameters, $\beta, b_{0}$ and $o_{0}$ have been heuristically optimized with acquired data from 10 tests. In this paper, the Nelder-Mead Simplex method is applied for optimizing the error model parameters, because this method commonly used in non-linear optimization.

\section{Experiments}

The accuracy of this orientation estimation is tested with the measurement data from the test bench of this application. The results from this orientation estimation are compared with the rotating angles measured by the encoder of the test bench. In brief, the test data are acquired from a CRM100 pin-point gyroscope with 20 measurement data.

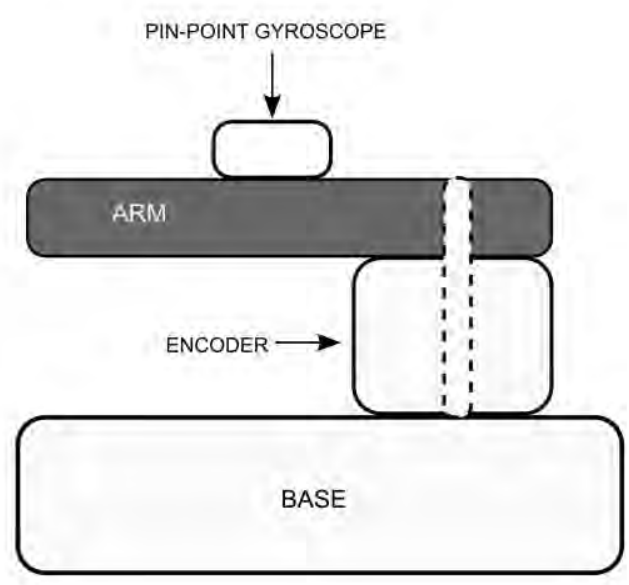

Fig. 2. Test bench

The test bench is composed of a base, an arm and an encoder as shown in Fig [X2]. The length of the wooden arm is 80 centimeter and is fixed with the shaft that connects to the encoder. The gyroscope is mounted at the center of the top side of the arm.

The measurement data are acquired while the arm is manually rotated $90^{\circ}$ clockwise and counter-clockwise with different speed. Therefore, the measurement data are not uniform. Then, the results from the orientation estimation are independent on the rotating velocity of the arm.

\section{Results}




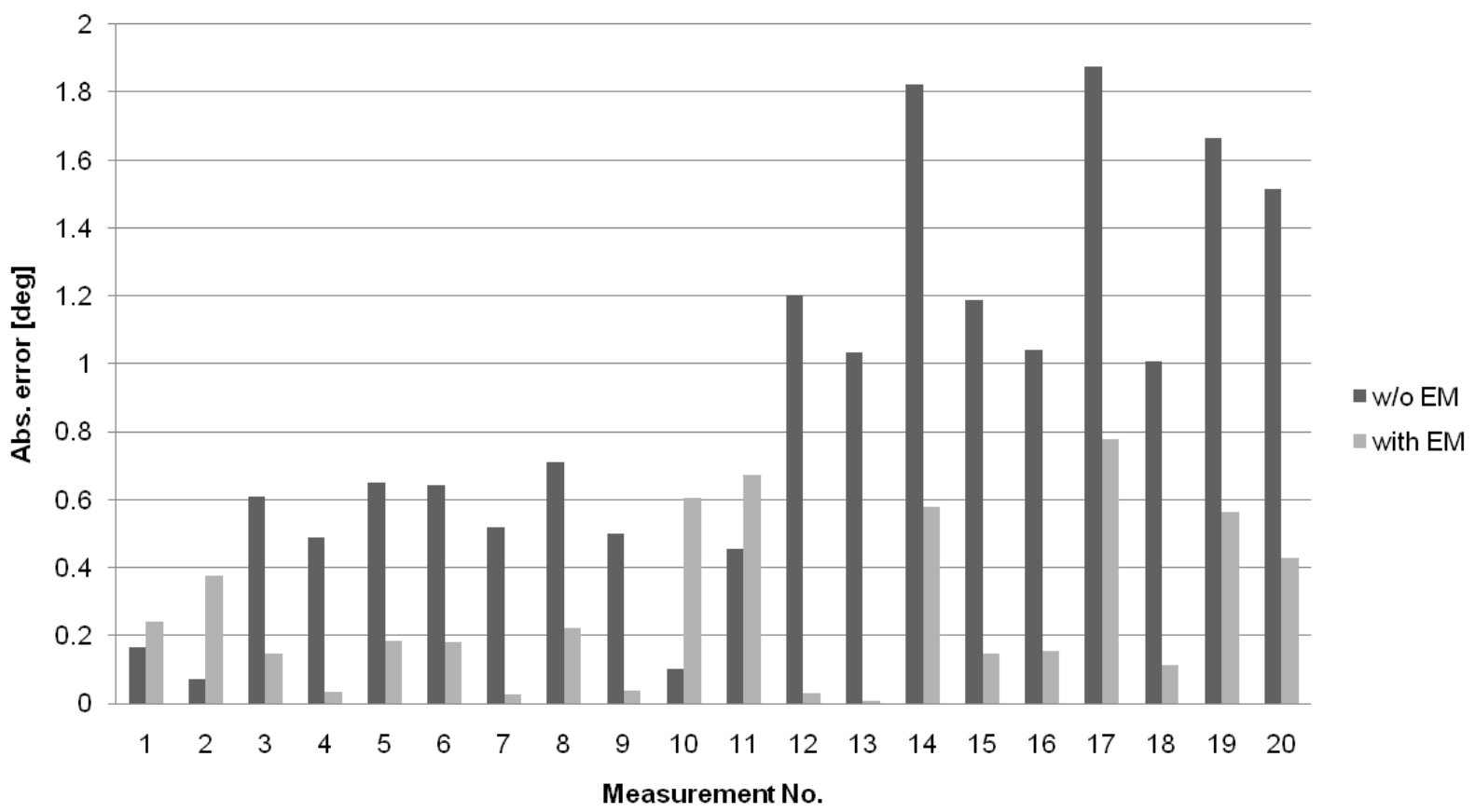

Fig. 3. Maximum absolute error from the orientation estimation

(a)

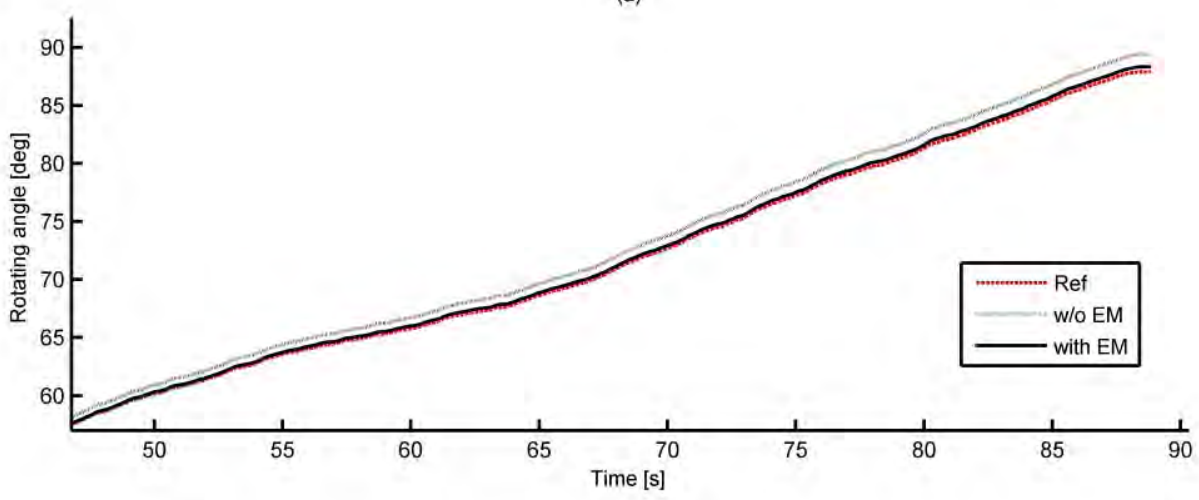

(b)

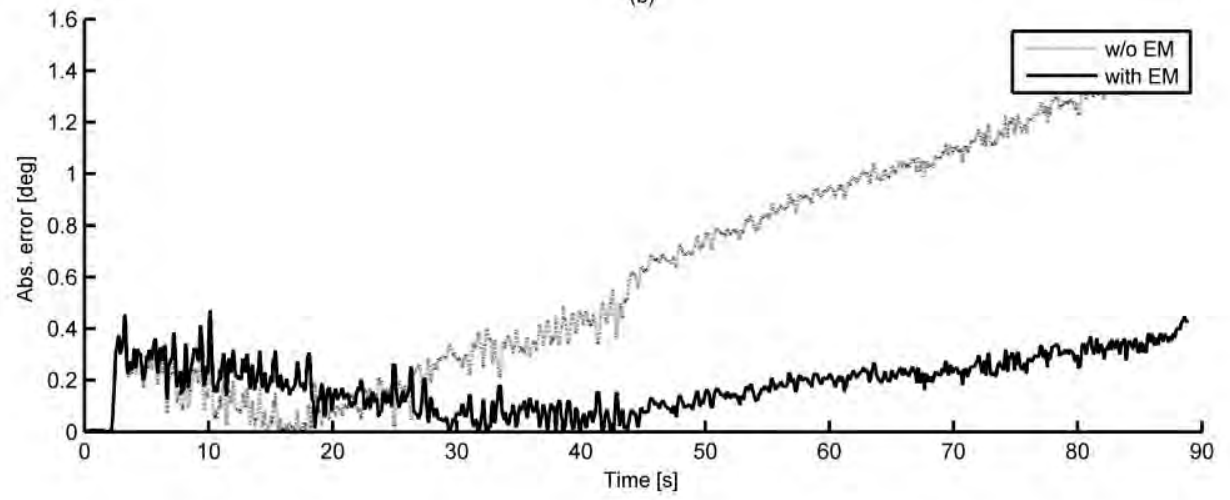

Fig. 4. (a) Orientation estimation of the measurement No. 20, (b) Absolute error of the estimation from measurement No. 20

As Fig. 3, the results show that the orientation estimation with help of error model (EM) is significantly more accurate than one without error model. The maximum absolute error of the orientation estimation without EM is more than $1.8^{\circ}$ within 90 second. With help of EM, the maximum absolute error is less than $0.8^{\circ}$ within 90 second. Although, there are some measurement data that the estimations without $\mathrm{EM}$ are more accurate than one with EM, the overall performance of the 1D-orientation with help of EM is better than one without EM. 
The EM reduces the error from sensor drift that accumulates over time. As Fig. 4(b), the error from the estimation without EM increases over time. But the error from the estimation with EM is suppressed and increases over time slower than one without EM. It shows that the implementation of the error model module is successfully improves the performance of the orientation estimation.

\section{Conclusion and Outlook}

The error model based on shaping filter technique significantly improves the accuracy of 1D-orientation estimation with the measurement data from the test bench. With parameter optimization, the error from the orientation estimation with the error model is less than $1^{\circ}$ within 90 second.

For further improvement, the inertial measurement unit (IMU) will be applied for this application. With help of IMU, it is possible to determine 3D-orientation of an object. Therefore, the flexibility and reliability of the orientation estimation for an intelligent wrench will be increased.

\section{References}

[1] M. Haid, T. Dorf, G. Marquardt, and U. Schneider, Orientation Tracking with Low cost Sensors as a Kalman Filter Application, IAIN 2003, World Congress, Berlin (2003);

[2] S. H. Won, F. Golnaraghi, and W. Melek, A Fastening Tool Tracking System Using an IMU and a Position Sensor with Kalman Filters and a Fuzzy Expert System, Industrial Electronics, IEEE Transactions on, vol. 56, no. 5, pp. 1782 1792, (2009);

[3] M. Haid, Improvement of the Referenceless Inertial Objeckt-Tracking for Low-cost IndoorNavigation by Kalman-filtering (Verbesserung der referenzlosen inertialen Objecktverfolgung zur low-cost Indoor-navigaton durch Anwendung der Kalman-filterung), Ph.D. dissertation, Universität Siegen.(2004);

[4] E. Foxlin, Inertial Head-tracker Sensor Fusion by a Complimentary Separate-bias Kalman Filter, Virtual Reality Annual International Symposium, vol. 0, p. 185, (1996);

[5] B. Friedland, Treatment of Bias in Recursive Filtering, Automatic Control, IEEE Transactions on, vol. 14, no. 4, pp. 359 - 367, Aug 1969

[6] R. Zhu, and Z. Zhou, A Small Low-cost Hybrid Orientation System and its Error Analysis," Sensors Journal, IEEE, vol. 9, no. 3, pp. 223 230, (2009);

[7] Y. Xiaoping, E. Bachmann, and R. McGhee, A Simplified Quaternion Based Algorithm for Orientation Estimation from Earth Gravity and
Magnetic Field Measurements, Instrumentation and Measurement, IEEE Transactions on, vol. 57 , no. 3, pp. $638-650$, ( 2008);

[8] L. Zhao, Q. Nie, and Q. Guo, Unscented Kalman Filtering for SINS Attitude Estimation, in Control and Automation, 2007. ICCA 2007. IEEE International Conference on, pp. 228 -232, (2007);

[9] M. Romanovas, L. Klingbeil, M. Trachtler, and Y. Manoli, Efficient Orientation Estimation Algorithm for Low cost Inertial and Magnetic Sensor Systems, in Statistical Signal Processing, 2009. SSP '09. IEEE/SP 15th Workshop on, pp. 586 589.(2009);

[10] P. Martin, and E. Salaun, Invariant Observers for Attitude and Heading Estimation from Low-cost Inertial and Magnetic Sensors, in Decision and Control, 2007 46th IEEE Conference on, pp. 1039 -1045, (2007);

[11] J. Marins, X. Yun, E. Bachmann, R. McGhee, and M. Zyda, An Extended Kalman Filter for Quaternion-based Orientation Estimation Using MARG Sensors," in Intelligent Robots and Systems, 2001. Proceedings. 2001 IEEE/RSJ International Conference on, vol. 4, pp. 2003 2011 vol.4, (2001);

[12] H. Rehbinder and X. Hu, Drift-free Attitude Estimation for Accelerated Rigid Bodies, Automatica, vol. 40, no. 4, pp. 653 - 659, (2004);

[13] X. Kong, INS Algorithm Using Quaternion Model for Low cost IMU, Robotics and Autonomous Systems, vol. 46, no. 4, pp. 221 - 246, 2004.

[14] S. Julier, J. Uhlmann, and H. Durrant-Whyte, A new Approach for Filtering Nonlinear Systems, in American Control Conference, 1995.

Proceedings of the, vol. 3, pp. $1628-1632$ vol.3. (1995);

[15] W. Dayawansa, and C. Martin, A Converse Lyapunov Theorem for a Class of Dynamical Systems which Undergo Switching, Automatic Control, IEEE Transactions on, vol. 44, no. 4, pp. 751 -760, (1999);

[16] J.C. Lagarias, J. A. Reeds, M. H. Wright, and P. E. Wright, Convergence Properties of the NelderMead Simplex Method in Low Dimensions, SIAM Journal of Optimization, Vol. 9 Number 1,112-147 (1998)

[17] S. Maybeck, Stochastic Models, Estimation, and Control" Volume 1, ACADEMIC PRESS, New York, 181-193, (1979):

[18] O. Loffeld, Estimation Theory II, Kalman-filter Application (Estimationstheorie II, Anwendungen Kalman - Filter). Munich, Vienna: R. OldenbourgVerlag, (1990); 